\title{
Coloring Uniform Hypergraphs with Small EDGE DEGREES
}

\author{
ALEXANDR V. KOSTOCHKA*, MOHIT KUMBHAT and \\ VOJTĚCH RÖDL ${ }^{\dagger}$
}

Dedicated to the 60th birthday of László Lovász

Let $k$ be a positive integer and $n=\left\lfloor\log _{2} k\right\rfloor$. We prove that there is an $\varepsilon=\varepsilon(k)>$ 0 such that for sufficiently large $r$, every $r$-uniform hypergraph with maximum edge degree at most

$$
\varepsilon(k) k^{r}\left(\frac{r}{\ln r}\right)^{\frac{n}{n+1}}
$$

is $k$-colorable.

\section{INTRODUCTION}

The degree of an edge $e$ in a hypergraph $G$ is the number of other edges of $G$ intersecting $e$, and the maximum edge degree of $G$ is the maximum over the degrees of its edges. A natural question is: Which bound on the maximum edge degree of an $r$-uniform hypergraph $G$ provides that $G$ is $k$-colorable? The classical result in this direction belongs to Erdős and Lovász. In their seminal paper [2] (where the Lovász Local Lemma appeared), they proved the following bound.

\footnotetext{
${ }^{*}$ Research of this author is supported in part by NSF grant DMS-0650784 and by grant 06-01-00694 of the Russian Foundation for Basic Research.

${ }^{\dagger}$ Research of this author was supported by NSF grants DMS-0300529 and DMS0800070 .
} 
Theorem 1 [2]. If $k, r \geq 2$, then every $r$-uniform hypergraph with maximum edge degree at most $\frac{1}{4} k^{r}$ is $k$-colorable.

The proof works also for list coloring. A remarkable feature of this result is that it works for all $k, r \geq 2$, and in many cases the bound is rather close to the best possible. In particular, Erdős and Lovász [2] showed that the bound cannot be significantly improved even if we consider only hypergraphs with high girth. If we denote by $D(k, r)$ (respectively, $D(k, r, g)$ ) the minimum $D$ such that there exists an $r$-uniform non- $k$-colorable hypergraph $G$ with (girth at least $g$ and) maximum edge degree $D$, then the results mentioned above can be summarized as follows. For every $g \geq 2$,

$$
\frac{1}{4} k^{r}<D(k, r) \leq 20 r^{3} k^{r-1} \text {. }
$$

The upper bound in (1) was recently improved for $k<r$ by Kostochka and Rödl [5] to $r\left\lceil r k^{r-1} \ln k\right\rceil$.

Let $m(r, k)$ denote the minimum number of edges in an $r$-uniform hypergraph that is not $k$-colorable. Elaborating the proof of a lower bound on $m(r, 2)$, and using the Local Lemma, Radhakrishnan and Srinivasan [6] improved the lower bound on $D(2, r)$ for large $r$ to

$$
D(2, r) \geq 0.17 \cdot 2^{r} \sqrt{r / \ln r} .
$$

The main result of this paper is the following extension of (2) to fixed $k$ and large $r$.

Theorem 2. For every integer $k \geq 2$, let $\varepsilon=\varepsilon(k)=\exp \left\{-4 k^{2}\right\}$ and $n=n(k)=\left\lfloor\log _{2} k\right\rfloor$. Then for every sufficiently large $r$, every $r$-uniform hypergraph with maximum edge degree at most $D=\varepsilon k^{r}\left(\frac{r}{\ln r}\right)^{\frac{n}{n+1}}$ is $k$ colorable. In other words, $D(k, r)>\varepsilon k^{r}\left(\frac{r}{\ln r}\right)^{\frac{n}{n+1}}$.

Recall that for the class of simple hypergraphs, Kostochka and Kumbhat [4] proved recently a slightly stronger bound than Theorem 2 gives. Recall that a hypergraph $G$ is b-simple if $\left|e \cap e^{\prime}\right| \leq b$ for every distinct $e, e^{\prime} \in E(G)$.

Theorem 3 [4]. If $b \geq 1, k \geq 2$, and $\varepsilon>0$ are fixed and $r$ is sufficiently large, then every $r$-uniform $b$-simple hypergraph $G$ with maximum edge degree at most $k^{r} r^{1-\varepsilon}$ is $k$-colorable. 
We think that the statement of Theorem 3 holds for all $r$-uniform hypergraphs.

The proof of Theorem 2 uses the Local Lemma, ideas of Radhakrishnan and Srinivasan [6], and the proof of the lower bound on $m(r, k)$ by Kostochka [3].

The structure of the paper is as follows. In the next section, a semirandom procedure Evolution is described and some of its simple properties are derived. In Section 3 we study the structure of so called cause trees arising in the analysis of Evolution. In the next two sections we define some auxiliary "bad" events and estimate their probabilities. Using the independence structure of these auxiliary events and the Local Lemma, in the final section we show that for hypergraphs satisfying the conditions of Theorem 2, with positive probability Evolution gives a proper $k$-coloring. This means that such a coloring exists.

\section{Coloring Procedure Evolution and its Properties}

Let $k, n$ and $\varepsilon$ be as in the statement of the theorem. Let

$$
r \geq \exp \left\{2 \varepsilon^{-2(n+1)}\right\} .
$$

Throughout the paper we will use the notation

$$
z=\left\lfloor 4 k^{2} r / \ln r\right\rfloor .
$$

Fix some $0<p<2^{-k^{r} r}$. Then there is the unique positive integer $s$ such that $s p \leq \frac{\ln r}{(n+1) r}<(s+1) p$. Let $G=(V, E)$ be an $r$-uniform hypergraph with maximum edge degree at most $D=\varepsilon k^{r}\left(\frac{r}{\ln r}\right)^{\frac{n}{n+1}}$.

The coloring procedure Evolution described below consists of $n+1$ stages, and every stage apart from Stage 0 consists of $s$ steps. For $1 \leq l \leq n$ and $1 \leq i \leq s$, Step $(l-1) s+i$ is the $i$ th step in Stage $l$.

We also fix a linear order $L$ on $V(G)$. Now, the procedure works as follows.

Stage 0. (O1) Color every vertex $v \in V(G)$ randomly and independently, with a color $\phi(v) \in\{0,1,2, \ldots, k-1\}$ chosen uniformly in this set. 
(O2) For every $v \in V(G)$, define the random variable $I(v)$ with the values in $\{1,2, \ldots, s n\} \cup\{\infty\}$ as follows:

$$
\operatorname{Pr}\{I(v)=x\}= \begin{cases}p, & \text { if } x \in\{1,2, \ldots, s n\} \\ 1-p s n, & \text { if } x=\infty\end{cases}
$$

Each random variable $I(v)$ is defined to be mutually independent of all other $I(w)$.

Stage $l, l=1, \ldots, n$.

STEP $i+s(l-1), 1 \leq i \leq s$. Following order $L$, for one by one vertex $v \in V(G)$, check whether

(C1) $I(v)=(l-1) s+i$ and

(C2) $v$ belongs to an edge that was monochromatic, say, of color $\alpha$, before Stage $l$, and still is monochromatic at the current moment.

If both conditions $(\mathrm{C} 1)$ and $(\mathrm{C} 2)$ hold, then recolor $v$ with color $\alpha+2^{l-1}$ (modulo $k$ ). Otherwise, do nothing with $v$.

Remark 1. By Condition (C1), each vertex can be recolored at most once.

Remark 2. As it follows from the description of the procedure, every step consists of $|V(G)|$ smaller steps (one per vertex).

Lemma 1. For every $w, q \geq 1$, every set $W \subseteq V$ with $|W|=w$, and every set $Q \subseteq\{1,2, \ldots, s n\}$ with $|Q|=q$, the probability that for each vertex $v \in W, I(v) \in Q$ is at most $(q p)^{w}$.

Proof. For every vertex $v \in V(G)$ and every $1 \leq l \leq n$ and $1 \leq i \leq s$, $\operatorname{Pr}\{I(v)=s(l-1)+i\}=p$. Therefore, the probability that $I(v) \in Q$ is at most $q p$. The mutual independence of all $I(v)$ yields the lemma.

For an edge $e \in E$ and $1 \leq l \leq n$, let

$$
M(e, l)=\{v \in e: I(v) \leq s l\} .
$$

Lemma 2. For every $e \in E$ and $1 \leq l \leq n$,

$$
\operatorname{Pr}\{|M(e, l)| \geq z\} \leq \varepsilon^{0.5 r} .
$$


Proof. It is enough to prove the lemma for $l=n$. Observe that $|M(e, n)| \geq$ $z$ means that there exists a set $Z \subset e$ with $|Z|=z$ such that $I(v) \leq s n$ for every $v \in Z$. Hence by Lemma 1 applied with $Q=[s n]$ and $W=Z$, this probability is at most

$$
\left(\begin{array}{l}
r \\
z
\end{array}\right)(n s p)^{z} \leq\left(\frac{e r}{z}\right)^{z}\left(\frac{n \ln r}{(n+1) r}\right)^{z} \leq\left(\frac{n e \ln r}{z(n+1)}\right)^{z} .
$$

Since $r$ is large and $z=\left\lfloor 4 k^{2} r / \ln r\right\rfloor>n$,

$$
\frac{n e \ln r}{z(n+1)} \leq \frac{e \ln r}{z+1} \leq \frac{e \ln ^{2} r}{4 k^{2} r} \leq r^{-0.6}
$$

Thus

$$
\left(\frac{n e \ln r}{z(n+1)}\right)^{z} \leq\left(r^{-0.6}\right)^{\left(4 k^{2} r / \ln r\right)-1}<e^{-2 k^{2} r}=\varepsilon^{0.5 r}
$$

Lemma 3. If a vertex is of color $\alpha$ at the end of Stage $l, l \geq 1$, then at the end of Stage 0 it can be colored only with colors $\alpha, \alpha-2^{0}, \alpha-2^{1}, \ldots$, $\alpha-2^{l-1}$ (modulo $\left.k\right)$.

Proof. By Remark 1, every vertex can be recolored at most once and by definition, a vertex of color $\beta$ can be recolored during Stage $j$ only with color $\beta+2^{j-1}$ (modulo $k$ ).

Definition [Blaming edges]. If an edge $e_{0}$ becomes monochromatic of color $\alpha$ during Stage $l$, then it must contain at the end of Stage 0 a vertex of color $\alpha-2^{l-1}$. Suppose that at the end of Stage 0 it contained vertices of colors $\alpha-2^{l_{1}-1}, \ldots, \alpha-2^{l_{h}-1}$, where $l_{h}=l$ and $l_{1}<l_{2}<\cdots<l_{h}$. Then for every $1 \leq j \leq h$, there exists an edge $e_{j}$ and a vertex $v_{j} \in e_{0} \cap e_{j}$ such that

(a) $e_{j}$ was monochromatic of color $\alpha-2^{l_{j}-1}$ at the end of Stage $l_{j}-1$;

(b) $v_{j}$ was recolored with $\alpha$ during Stage $l_{j}$ and it was the last vertex of this color in $e_{0}$ recolored with $\alpha$.

In this case we say that $e_{0}$ and $v_{j} l_{j}$-blame $e_{j}$.

Remark 3. If an edge $e_{0}$ becomes monochromatic of color $\alpha$ during Stage $l$, then all vertices of color $\alpha-2^{l-1}$ in $e_{0}$ change their colors to $\alpha$ during this stage. But since in every step, the vertices of $G$ are considered consecutively, there is the last vertex $v^{*}$ of color $\alpha-2^{l-1}$ in $e_{0}$ that changes its color. At that moment, any monochromatic edge $e$ of color $\alpha-2^{l-1}$ containing $v^{*}$ shares only $v^{*}$ with $e_{0}$. 
Remark 4. It may happen that an edge $e_{0}$ can blame more than one edge containing the same vertex $v_{j}$. On the other hand, by definition, $e_{0}$ cannot blame an edge containing another vertex $v \in e_{0}$ with $\phi(v)=\phi\left(v_{j}\right)$.

Definition [Cause trees]. If an edge $e_{0}$ is monochromatic of color $\alpha$ at the end of Stage $l$, then a cause tree $T=T\left(e_{0}, \alpha, l\right)$ is a subset of edges of $G$ defined by induction on $l$ as follows:

(a) The set $T$ always contains $e_{0}$.

(b) If $e_{0}$ was monochromatic of color $\alpha$ already after Stage 0 , then $T=\left\{e_{0}\right\}$ for every $l$.

(c) Suppose that at the end of Stage 0 edge $e_{0}$ contained vertices of colors $\alpha-2^{l_{1}-1}, \ldots, \alpha-2^{l_{h}-1}$, where $l_{h} \leq l$ and $l_{1}<l_{2}<\cdots<l_{h}$. Suppose further that for $j=1, \ldots, h$, edge $e_{0} l_{j}$-blames edge $e_{j}$. Then

$$
T=T\left(e_{0}, \alpha, l\right)=\left\{e_{0}\right\} \cup \bigcup_{j=1}^{h} T\left(e_{j}, \alpha-2^{l_{j}-1}, l_{j}-1\right) .
$$

Remark 5. By Remark 4 and the definition of cause trees, it could be that in the same outcome of Evolution for the same triple $\left(e_{0}, \alpha, l\right)$, we can construct several distinct cause trees $T=T\left(e_{0}, \alpha, l\right)$.

Definition [Levels of edges]. If $T=T\left(e_{0}, \alpha, l\right)$ is defined as above, then we also say that $e_{1}, e_{2}, \ldots, e_{h}$ are the edges of level 1 of $T$, the edges blamed by the edges of level 1 are the edges of level 2 of $T$, and so on. Thus, if an edge $e$ of a cause tree has vertices of exactly $t$ distinct colors at the end of Stage 0 , then $e$ blames either $t-1$ or $t$ other edges.

\section{Structure of Cause Trees}

Since each vertex can be recolored at most once, each edge at different stages of Evolution can become monochromatic with at most two colors. Furthermore, if an edge $e$ was monochromatic of a color $\alpha_{1}$ after Stage $l_{1}$ and becomes monochromatic of a color $\alpha_{2} \neq \alpha_{1}$ after Stage $l_{2}$, then $e$ has to be monochromatic of color $\alpha_{1}$ already after Stage 0 and all vertices of $e$ change their color to $\alpha_{2}=\alpha_{1}+2^{l_{2}-1}$ at Stage $l_{2}$. In this case, each cause tree for $e$ considered after Stage $l_{2}$ has exactly one edge of level 1 . 
In view of this, if an edge $e$ becomes monochromatic exactly once during Evolution, then the corresponding color $\alpha$ is called the main color of $e$ and denoted by $\mu(e)$, and if $e$ becomes monochromatic twice, then the main color of $e, \mu(e)$, is the first of these two colors.

If $e$ is monochromatic of some color $\alpha$ after some Stage $l$, then we say that $e$ is an l-unlucky edge.

Lemma 4. If $e_{0}$ is an l-unlucky edge with a cause tree $T$, then the main colors of all the edges of $T$ are distinct.

Proof. If $e$ and $e^{\prime}$ are edges of $T$, then there exist two sequences $e_{0}, e_{1}, \ldots$, $e_{q}=e$ and $e_{0}^{\prime}=e_{0}, e_{1}^{\prime}, \ldots, e_{q^{\prime}}^{\prime}=e^{\prime}$ such that $e_{j} l_{j}$-blames $e_{j+1}$ for $j=$ $0,1, \ldots, q-1$ and $e_{j}^{\prime} l_{j}^{\prime}$-blames $e_{j+1}^{\prime}$ for $j=0,1, \ldots, q^{\prime}-1$. Furthermore, $l_{0}>l_{1}>\cdots>l_{q-1}, l_{0}^{\prime}>l_{1}^{\prime}>\cdots>l_{q-1}^{\prime}$, and the sequences $l_{0}, l_{1}, \ldots, l_{q}$ and $l_{0}^{\prime}, l_{1}^{\prime}, \ldots, l_{q}^{\prime}$ are not identical. Thus, the numbers $2^{l_{0}-1}+2^{l_{1}-1}+\cdots+2^{l_{q-1}-1}$ and $2^{l_{0}^{\prime}-1}+2^{l_{1}^{\prime}-1}+\cdots+2^{l_{q^{\prime}-1}^{\prime}-1}$ are distinct and differ by less than $k$. On the other hand, by definition, the main color of $e$ is $\alpha-2^{l_{0}-1}-2^{l_{1}-1}-\cdots-2^{l_{q-1}-1}$ and the main color of $e^{\prime}$ is $\alpha-2^{l_{0}^{\prime}-1}-2^{l_{1}^{\prime}-1}-\cdots-2^{l_{q^{\prime}-1}^{\prime}-1}$. This proves the lemma.

Lemma 5. Suppose that $e_{0}$ is an l-unlucky edge with a cause tree T. If $e$ and $e^{\prime}$ are edges of $T$ and neither of them blames the other, then $e$ and $e^{\prime}$ are disjoint.

Proof. Assume that $e$ and $e^{\prime}$ have a common vertex $v$ and both belong to $T$. Then there exist two sequences $e_{0}, e_{1}, \ldots, e_{q}=e$ and $e_{0}^{\prime}=e_{0}, e_{1}^{\prime}, \ldots, e_{q^{\prime}}^{\prime}=e^{\prime}$ such that $e_{j} l_{j}$-blames $e_{j+1}$ for $j=0,1, \ldots, q-1$ and $e_{j}^{\prime} l_{j}^{\prime}$-blames $e_{j+1}^{\prime}$ for $j=0,1, \ldots, q^{\prime}-1$. Furthermore, $l_{0}>l_{1}>\cdots>l_{q-1}, l_{0}^{\prime}>l_{1}^{\prime}>\cdots>l_{q-1}^{\prime}$.

Claim 1. $l_{q-1} \neq l_{q^{\prime}-1}^{\prime}$.

Proof of Claim. If $l_{q-1}=l_{q^{\prime}-1}^{\prime}$, then $e$ and $e^{\prime}$ both were monochromatic at the end of Stage $l_{q-1}-1$. But by Lemma 4, their main colors differ. This proves the claim.

Thus below we can assume that $l_{q-1}<l_{q^{\prime}-1}^{\prime}$. It follows that $e$ ceased to be monochromatic before $e^{\prime}$ did. In particular, the vertex $v \in e \cap e^{\prime}$ was recolored from $\mu(e)$ to $\mu\left(e^{\prime}\right)$. This yields that

$$
\mu\left(e^{\prime}\right)-\mu(e)(\text { modulo } k \text { ) is a power of } 2 \text {. }
$$

Claim 2. $\mu\left(e^{\prime}\right)-\mu(e)=2^{l_{q-1}-1}$ modulo $k$. 
Proof of Claim. Recall that

$$
\begin{aligned}
& \mu\left(e^{\prime}\right)-\mu(e)=\left(\alpha-2^{l_{0}^{\prime}-1}-2^{l_{1}^{\prime}-1}-\cdots-2^{l_{q^{\prime}-1}^{\prime}-1}\right) \\
& -\left(\alpha-2^{l_{0}-1}-2^{l_{1}-1}-\cdots-2^{l_{q-1}-1}\right) .
\end{aligned}
$$

In this expression, $\alpha$ cancels out and every other summand apart from $2^{l_{q-1}-1}$ is divisible by $2^{l_{q-1}}$. Together with (7), this yields the claim.

Claim 2 implies that $v$ was recolored during Stage $l_{q-1}$ and thus $\mu\left(e^{\prime}\right)=$ $\mu\left(e_{q-1}\right)$. This contradicts Lemma 4 .

Lemma 6. Let $e_{0}$ be an edge of $G$ that is unlucky after Stage l. Let $T$ be a cause tree for this event such that for every pair of edges $\left(e_{1}, e_{2}\right)$ of $T$ such that $e_{1}$ blames $e_{2}$, we have $\mu\left(e_{1}\right)-\mu\left(e_{2}\right) \in\left\{1,2, \ldots, 2^{l-1}\right\}$ (modulo $k$ ). Then $|E(T)| \leq 2^{l}$. In particular, each cause tree has at most $2^{n} \leq k$ edges.

Proof. If $e_{1} l_{1}$-blames $e_{2}$ and $e_{2} l_{2}$-blames $e_{3}$, then $l_{2}<l_{1}$. Thus, under conditions of the lemma, for the root $e_{0}$ and an arbitrary edge $e$ of the tree, we have

$$
\mu\left(e_{0}\right)-\mu(e) \in\left\{1,2, \ldots, 2^{l-1}+2^{l-2}+\cdots+1\right\}=\left\{1,2, \ldots, 2^{l}-1\right\} .
$$

Now, Lemma 4 implies that $T$ has at most $1+\left(2^{l}-1\right)$ edges.

Below we will analyze which subsets of edges of $G$ can form cause trees $T(e, \alpha, l)$ for some values of $e, \alpha$ and $l$. Lemma 5 implies that every cause tree $T=T(e, \alpha, l)$ is an $r$-uniform hypergraph tree in the ordinary sense rooted at $e$. Moreover, every vertex of such a tree belongs to at most two edges of this tree. By Lemma 6 , such a tree has at most $k$ edges. In connection with this, let us fix some notation.

Definition $[r$-Trees and sub- $r$-trees]. When we say " $r$-tree", we mean an $r$ uniform hypergraph tree with at most $k$ edges in which every vertex belongs to at most two edges of this tree. By a sub-r-tree of $G$ we mean an $r$-tree that is a subhypergraph of $G$.

Often, we will consider rooted $r$-trees. The root of an $r$-tree will be an edge of this $r$-tree, and not a vertex. Given an $r$-tree $T$ with a root $e_{0}$, the children of $e_{0}$ are the edges adjacent to $e_{0}$, and for $e \in E(T)$ at distance $d$ from $e_{0}$ (in $T$ ), the children of $e$ are the edges adjacent to $e$ that are at distance $d+1$ from $e_{0}$. Naturally, the descendants of an $e \in E(T)$ are its children, children of children and so on. If $e_{1}$ is a descendant of $e_{2}$, then $e_{2}$ is an ancestor of $e_{1}$. For an $r$-tree $T$ with a root $e_{0}$ and another edge $e_{1}$ of $T$, by $T\left(e_{1}\right)$ we denote the subtree of $T$ formed by $e_{1}$ and all its descendants. We will use the following fact on sub- $r$-trees of $r$-uniform hypergraphs. 
Lemma 7. Let $H$ be an $r$-uniform hypergraph with maximum edge degree at most $D$. Let $e_{0} \in E(H)$. Then $e_{0}$ belongs to at most $(4 D)^{y-1}$ sub-r-trees of $H$ with $y$ edges.

Proof. Let $T$ be a sub- $r$-tree of $H$ containing $e_{0}$ with $|E(T)|=y$. Consider $T$ as a rooted $r$-tree with root $e_{0}$. Order the edges of $T e_{0}, e_{1}, \ldots, e_{y-1}$ starting from $e_{0}$ using Breadth-First search. We say that $T$ has type $\left(h_{0}, \ldots, h_{y-2}\right)$ if for $i=0, \ldots, y-2$, edge $e_{i}$ has exactly $h_{i}$ children. Since $h_{0}+\cdots+h_{y-2}=y-1$, the number of distinct types does not exceed the number of representation of $y-1$ as the sum of $y-1$ of ordered nonnegative summands, which equals $\left(\begin{array}{c}(y-1)+(y-1)-1 \\ y-2\end{array}\right)<4^{y-1}$. When we know the type of $T$, then for every edge $e_{i}, i \geq 1$, we know the immediate ancestor (father edge). So, we can embed a tree $T$ of a given type, edge by edge into $G$. Furthermore, at each step $i, i \geq 1$, we have at most $D$ choices for our edge among the edges of $G$ adjacent to its father edge. Thus, $e_{0}$ belongs to at most $D^{y-1} r$-trees of given type with $y$ edges. Since the number of distinct types is at most $4^{y-1}$, this proves the lemma.

\section{Auxiliary Events}

The goal of this section is to introduce the auxiliary events $\widetilde{W}\left(e_{0}, \alpha, T, l\right)$ that imply the "bad" events in Evolution and can be controlled. In the next section we estimate probabilities of these auxiliary events.

Definition [Events $W(e, \alpha, T, l)]$. Let $e \in E(G), \alpha \in[k], l \in\{1, \ldots, n\}$ and $T$ be a sub- $r$-tree of $G$ rooted at $e$. Then let $W(e, \alpha, T, l)$ be the event that edge $e$ is monochromatic of color $\alpha$ after Stage $l$ of Evolution, and a cause tree for this is $T$.

Lemma 8. Let $e \in E(G), \alpha \in[k], l \in\{1, \ldots, n\}$ and $T$ be a sub-r-tree of $G$ rooted at $e$. Also, let $e_{1}, \ldots, e_{q}$ be the edges of $T$ of the first level, i.e., the edges of $T$ sharing a vertex with $e$. For $j \in[q]$, let $e \cap e_{j}=\left\{v_{j}\right\}$. Let $Q \doteq\left\{v_{1}, \ldots, v_{q}\right\}$. If $W(e, \alpha, T, l)$ occurs, then the following properties hold:

(W1) For every $v \in e, \phi(v) \in\{\alpha\} \cup \phi(Q) \subseteq\left\{\alpha, \alpha-2^{0}, \alpha-2^{1}, \ldots, \alpha-2^{l-1}\right\}$ (modulo $k$ ).

(W2) For $j \in[q], \phi\left(v_{j}\right) \neq \alpha$, and for distinct $j$ and $j^{\prime}, \phi\left(v_{j^{\prime}}\right) \neq \phi\left(v_{j}\right)$. In particular, if $A_{j}=A_{j}(e, \phi)=\left\{v \in e: \phi(v)=\phi\left(v_{j}\right)\right\}$, then all sets $A_{j}$ are disjoint subsets of $e$. 
(W3) $I(v) \leq l s$ for each $v \in \bigcup_{j=1}^{q} A_{j}$.

Moreover, for each $j \in[q]$, if $v_{j}$ becomes of color $\alpha$ at Stage $l_{j}$, then

(W4) $\alpha-\phi\left(v_{j}\right)=2^{l_{j}-1}$;

(W5) the event $W\left(e_{j}, \phi\left(v_{j}\right), T\left(e_{j}\right), l_{j}-1\right)$ occurs;

(W6) for every $u \in e_{j}$ with $I(u)>\left(l_{j}-1\right) s$, we have also $I(u) \geq I\left(v_{j}\right)$; and

(W7) for each $u \in A_{j}-v_{j},\left(l_{j}-1\right) s+1 \leq I(u) \leq I\left(v_{j}\right)$.

(W8) If $e$ was already monochromatic after Stage $l-1$, then for each $v \in e$, $I(v) \notin[s(l-1)+1, s l]$.

Proof. We will prove (W1)-(W8) one by one.

(W1) holds by the definition of cause trees and Lemma 3.

By the definition of cause trees, for each $j \in[q], v_{j}$ is the last vertex of color $\phi\left(v_{j}\right)$ that changed its color to $\alpha$. This implies both statements of (W2).

Since each $v \in \bigcup_{j=1}^{q} A_{j}$ has changed its color by Stage $l$, by condition (C1) in the definition of Evolution, (W3) follows.

(W4) also follows from the definition of Evolution.

If $e_{j}$ were not monochromatic of color $\phi\left(v_{j}\right)$ after Stage $l_{j}-1$, then $v_{j}$ would not obtain color $\alpha$ blaming $e_{j}$. This yields (W5).

If some $u \in e_{j}$ would have $\left(l_{j}-1\right) s<I(u)<I\left(v_{j}\right)$, then by the definition of Evolution, it would mean that $u$ did not change its color before Stage $l_{j}$. Recall that by (W5) edge $e_{j}$ is monochromatic after Stage $l_{j}-1$. Thus if Condition (C2) holds at the moment $I(u)$, then vertex $u$ should change its color at this moment, i.e. earlier than $v_{j}$ did. If not, this means that some other vertex of $e_{j}$ has already changed its color. In both cases, $v_{j}$ would not blame $e_{j}$. This contradiction proves (W6).

Now (W7) follows from the facts that all vertices in $A_{j}$ must change their colors in Stage $l_{j}$ (in order to change it from $\phi\left(v_{j}\right)$ to $\alpha$ ) and that $v_{j}$ is the last vertex in $A_{j}$ that changes its color.

If $e$ were monochromatic after Stage $l-1$, and $I(v) \in[s(l-1)+1, s l]$ for some $v \in e$, then $v$ would change its color, and so $W(e, \alpha, T, l)$ would not happen. This proves (W8).

Unfortunately, events $W(e, \alpha, T, l)$ and $W\left(e^{\prime}, \alpha^{\prime}, T^{\prime}, l^{\prime}\right)$ can be dependent even if $V\left(T^{\prime}\right)$ is disjoint from $V(T)$. For example, some edge $e_{0} \in E(G)$ may intersect both $V(T)$ and $V\left(T^{\prime}\right)$. In this case, event $W(e, \alpha, T, l)$ may prompt a vertex $v \in e_{0} \cap V(T)$ to change its color. This in turn, can make 
$e_{0}$ monochromatic and so prompt a vertex $v^{\prime} \in e_{0} \cap V\left(T^{\prime}\right)$ to change its color, which may affect $W\left(e^{\prime}, \alpha^{\prime}, T^{\prime}, l^{\prime}\right)$.

Therefore, for each $e_{0} \in E(G)$, each sub-r-tree $T$ of $G$ with root $e_{0}$ and $|E(T)| \leq k$, and each color $\alpha$, we will introduce the auxiliary event $\widetilde{W}\left(e_{0}, \alpha, T, l\right)$ that contains the event $W\left(e_{0}, \alpha, T, l\right)$, and in addition essentially possesses properties (W1)-(W8) above, but does not depend on the values of $\phi(u)$ and $I(u)$ for all $u \notin V(T)$. This will imply that each $W(e, \alpha, T, l)$ is independent of all $\widetilde{W}\left(e_{0}^{\prime}, \alpha^{\prime}, T^{\prime}, l^{\prime}\right)$ with $V\left(T^{\prime}\right) \cap V(T)=\emptyset$.

We define these events by induction on the number of edges in $T$.

If $E(T)=\left\{e_{0}\right\}$, then the event $\widetilde{W}\left(e_{0}, \alpha, T, l\right)$ means that all of the following holds

(i) $\phi\left(e_{0}\right)$ is monochromatic of color $\alpha$,

(ii) $\left|M\left(e_{0}, n\right)\right|<z$, and

(iii) $I(v)>l s$ for every $v \in e_{0}$.

Suppose that the event $\widetilde{W}\left(e_{0}, \alpha, T, l\right)$ is defined for all parameters $e_{0}, \alpha$, $T, l$ such that $|E(T)|<y$. Let $e_{0} \in E(G), \alpha \in[k]$, and $T$ be any sub-r-tree $T$ of $G$ with root $e_{0}$ and $y$ edges. Let $e_{1}, \ldots, e_{q}$ be the edges of $T$ sharing a vertex with $e$. For $j \in[q]$, let $e \cap e_{j}=\left\{v_{j}\right\}$. Let $Q \doteq\left\{v_{1}, \ldots, v_{q}\right\}$. We say that $\widetilde{W}\left(e_{0}, \alpha, T, l\right)$ occurs, if either $|M(e, n)| \geq z$ for at least one $e \in E(T)$ or all of the following holds:

$(\widetilde{W} 1)$ For every $v \in e, \phi(v) \in\{\alpha\} \cup \phi(Q) \subseteq\left\{\alpha, \alpha-2^{0}, \alpha-2^{1}, \ldots, \alpha-2^{l-1}\right\}$ (modulo $k)$.

$(\widetilde{W} 2)$ For $j \in[q], \phi\left(v_{j}\right) \neq \alpha$, and for distinct $j$ and $j^{\prime}, \phi\left(v_{j^{\prime}}\right) \neq \phi\left(v_{j}\right)$. In particular, if $A_{j}=A_{j}(e, \phi)=\left\{v \in e: \phi(v)=\phi\left(v_{j}\right)\right\}$, then all sets $A_{j}$ are disjoint.

$(\widetilde{W} 3) I(v) \leq l s$ for each $v \in \bigcup_{j=1}^{q} A_{j}$.

Moreover, for each $j \in[q]$, if $\left(l_{j}-1\right) s+1 \leq I\left(v_{j}\right) \leq s l_{j}$, then

$(\widetilde{W} 4) \alpha-\phi\left(v_{j}\right)=2^{l_{j}-1}$;

$(\widetilde{W} 5)$ event $\widetilde{W}\left(e_{j}, \phi\left(v_{j}\right), T\left(e_{j}\right), l_{j}-1\right)$ occurs;

$(\widetilde{W} 6)$ for every $u \in e_{j}$ with $I(u)>\left(l_{j}-1\right) s$, we have also $I(u) \geq I\left(v_{j}\right)$, and $(\widetilde{W} 7)$ for each $u \in A_{j}-v_{j},\left(l_{j}-1\right) s+1 \leq I(u) \leq I\left(v_{j}\right)$.

$(\widetilde{W} 8)$ If event $\widetilde{W}\left(e_{0}, \alpha, T, l-1\right)$ occurs, then for each $v \in e_{0}, I(v) \notin$ $[s(l-1)+1, s l]$.

The following two lemmas justify the introduction of the events $\widetilde{W}(e, \alpha, T, l)$. 
Lemma 9. Let $e_{0} \in E(G), \alpha \in[k], l \in\{0, \ldots, n\}$ and $T$ be a sub-r-tree of $G$ with root $e_{0}$. If the event $W\left(e_{0}, \alpha, T, l\right)$ occurs, then the event $\widetilde{W}\left(e_{0}, \alpha, T, l\right)$ also occurs.

Proof. Suppose that for some values of the parameters $e_{0}, T, l$, and $\alpha$, $W\left(e_{0}, \alpha, T, l\right)$ occurs but $\widetilde{W}\left(e_{0}, \alpha, T, l\right)$ does not occur. We may assume that $l$ is minimal with this property, i.e., that for all quadruples $\left(e_{0}^{\prime}, \alpha^{\prime}, T^{\prime}, l^{\prime}\right)$ with $l^{\prime}<l$ the lemma holds.

Let us check which of the properties in the definition of $\widetilde{W}\left(e_{0}, \alpha, T, l\right)$ may fail. Since $(\widetilde{W} 1)$ and $(\widetilde{W} 2)$ coincide with (W1) and (W2), respectively, they hold. For the same reason, properties $(\widetilde{W} 4),(\widetilde{W} 6)$, and $(\widetilde{W} 7)$ hold. Property ( $\widetilde{W} 5)$ follows from (W5) and the minimality of our counterexample. Property $(\widetilde{W} 3)$ follows from the fact that otherwise, by the definition of Evolution (Condition (C1)), some vertex in $\bigcup_{j=1}^{q} A_{j}$ would not change its color to $\alpha$.

Assume finally that $(\widetilde{W} 8)$ does not hold, in other words, that $\widetilde{W}\left(e_{0}, \alpha, T\right.$, $l-1$ ) occurs, and for some $v \in e_{0}, I(v) \in[s(l-1)+1, s l]$. By (W8), this implies that $W\left(e_{0}, \alpha, T, l-1\right)$ does not occur, i.e., after Stage $l-1, e_{0}$ is not monochromatic of color $\alpha$. It follows that in order $e_{0}$ to become monochromatic of color $\alpha$ after Stage $l$, we need $I(u) \in[s(l-1)+1, s l]$ for some $u \in \bigcup_{j=1}^{q} A_{j}$. On the other hand, by $(\widetilde{W} 3)$ for the event $\widetilde{W}\left(e_{0}, \alpha, T\right.$, $l-1), I(u) \leq(l-1) s$ for each $u \in \bigcup_{j=1}^{q} A_{j}$. This contradiction finishes the proof of the lemma.

Lemma 10. Let $e_{0} \in E(G), \alpha_{0} \in[k], l_{0} \in\{0, \ldots, n\}$, and $T_{0}$ be a sub-rtree of $G$ with root $e_{0}$. Then $\widetilde{W}\left(e_{0}, \alpha_{0}, T_{0}, l_{0}\right)$ is independent of all events $\widetilde{W}(e, \alpha, T, l)$ such that $V(T) \cap V\left(T_{0}\right)=\emptyset$.

Proof. By definition, the events $\widetilde{W}\left(e_{0}, \alpha_{0}, T_{0}, l_{0}, \psi\left(e_{0}\right)\right)$ are completely defined when we know the values of $\phi(v)$ and $I(v)$ for all $v \in V\left(T_{0}\right)$. This yields the lemma.

\section{Probabilities of the Auxiliary Events}

The whole proof is based on the following lemma. 
Lemma 11. Let $D:=\varepsilon k^{r}\left(\frac{r}{\ln r}\right)^{\frac{n}{n+1}}$ and $G$ be an $r$-uniform hypergraph with maximum edge degree at most $D$. Let $e \in E(G), \alpha \in[k]$, and $0 \leq l \leq n$. Let $T$ be a rooted sub-r-tree of $G$ with root e. If $T$ has $y$ edges, then

$$
\operatorname{Pr}(\widetilde{W}(e, \alpha, T, l)) \leq \varepsilon D^{-y}\left(\frac{r}{\ln r}\right)^{\frac{n-l}{n+1}}
$$

Proof. We start from proving the (simple) auxiliary inequality

$$
D^{k} \varepsilon^{0.5 r} \leq \frac{\varepsilon}{100 k}
$$

By definition and (3),

$$
D=\varepsilon k^{r}\left(\frac{r}{\ln r}\right)^{\frac{n}{n+1}} \leq \frac{\varepsilon}{\ln r} k^{r} r \leq \frac{\varepsilon}{2 \varepsilon^{-2(n+1)}} k^{2 r} \leq \frac{\varepsilon}{2} \varepsilon^{4} k^{2 r} .
$$

Since $\varepsilon^{4}=e^{-16 k^{2}}<\frac{1}{100 k},(8)$ would follow from the fact that $\left(k^{2 r}\right)^{k} \varepsilon^{0.5 r} \leq 1$, which is equivalent to

$$
\left(k^{2 r}\right)^{k} \leq\left(e^{4 k^{2}}\right)^{0.5 r} .
$$

The last inequality is obvious.

To prove the lemma, we use induction on $l$. Consider first $l=0$. If $\widetilde{W}(e, \alpha, T, 0)$ occurs, then by $(\widetilde{W} 1), \phi(v)=\alpha$ for each $v \in e$. Thus, in this case

$$
\operatorname{Pr}(\widetilde{W}(e, \alpha, T, 0))=k^{-r}=\frac{\varepsilon}{D}\left(\frac{r}{\ln r}\right)^{\frac{n}{n+1}} .
$$

This proves the case $l=0$.

Now, suppose that the lemma holds for every $l^{\prime}<l$. Consider the event $\widetilde{W}(e, \alpha, T, l)$ for some $e \in E(G)$, an $r$-tree $T$ with $y$ edges rooted at $e$, and $\alpha \in[k]$.

Let $X(T)$ denote the event that for at least one $e^{\prime} \in E(T)$, the set $M\left(e^{\prime}, n\right)=\left\{v \in e^{\prime}: I(v) \leq s n\right\} \quad\left(\mathrm{cf}\right.$. (6)) has at least $z=\left\lfloor 4 k^{2} r / \ln r\right\rfloor$ elements. More shortly,

$$
X(T)=\bigcup_{e^{\prime} \in E(T)}\left\{\left|M\left(e^{\prime}, n\right)\right| \geq z\right\} .
$$

Let $\overline{X(T)}$ denote the complement of $X(T)$. Suppose that the event $\widetilde{W}(e, \alpha, T, l) \cap \overline{X(T)}$ occurs. 
When a sub- $r$-tree $T$ with root $e$ is given, it automatically defines the edges $e_{1}, \ldots, e_{q}$ of $T$ that share a vertex with $e$. It also defines for every $j \in[q]$, the vertex $\left\{v_{j}\right\}=e \cap e_{j}$ and the number $y_{j}$ of edges in $T\left(e_{j}\right)$. Below, when $T$ is given, we will denote $Q=Q(T, e) \doteq\left\{v_{1}, \ldots, v_{q}\right\}$. Every outcome of Evolution such that $\widetilde{W}(e, \alpha, T, l) \cap \overline{X(T)}$ occurs defines the vector $\left(l_{1}, \ldots, l_{q}\right)$ such that $s\left(l_{j}-1\right)<I\left(v_{j}\right) \leq s l_{j}$ for each $j \in[q]$. By $(\widetilde{W} 3)$, for each $j \in[q], l_{j} \in[l]$. By $(\widetilde{W} 4)$ and $(\widetilde{W} 2)$, all $l_{j}$ are distinct.

Let $\Theta_{0}=\Theta_{0}(q, l)$ be the set of vectors $\left(l_{1}, \ldots, l_{q}\right)$ such that

$$
\text { all } l_{1}, \ldots, l_{q} \text { are distinct and belong to }[l] \text {. }
$$

By the previous paragraph,

$$
\widetilde{W}(e, \alpha, T, l) \cap \overline{X(T)}=\widetilde{W}(e, \alpha, T, l) \cap \overline{X(T)} \cap\left\{\left(l_{1}, \ldots, l_{q}\right) \in \Theta_{0}\right\} .
$$

Let $\Theta_{1}(q, l)=\Theta_{0}(q, l-1)$, i.e. the set of $\left(l_{1}, \ldots, l_{q}\right) \in \Theta_{0}$ such that $l_{j} \leq l-1$ for all $j \in[q]$. Let $\Theta_{2}=\Theta_{2}(q, l)=\Theta_{0}(q, l)-\Theta_{1}(q, l)$. For $i=1,2$, let

$$
F_{i}(e, \alpha, T, l)=\widetilde{W}(e, \alpha, T, l) \cap \overline{X(T)} \cap\left\{\left(l_{1}, \ldots, l_{q}\right) \in \Theta_{i}\right\} .
$$

By (11),

$$
\widetilde{W}(e, \alpha, T, l) \subseteq X(T) \cup F_{1}(e, \alpha, T, l) \cup F_{2}(e, \alpha, T, l)
$$

Our goal is to prove that for $i=1,2$,

$$
\operatorname{Pr}\left(F_{i}(e, \alpha, T, l)\right) \leq 0.4 \varepsilon D^{-y}\left(\frac{r}{\ln r}\right)^{\frac{n-l}{n+1}} .
$$

Since by Lemma 2 and (8), $\operatorname{Pr}(X(T)) \leq k \varepsilon^{0.5 r}<0.1 \varepsilon D^{-y}$, (12) and (13) will imply the lemma.

Observe that the condition " $l_{j} \leq l-1$ for all $j \in[q]$ " in the definition of $\Theta_{1}(\alpha, l)$ implies that if $\widetilde{W}(e, \alpha, T, l)$ occurs, then all conditions $(\widetilde{W} 1)-(\widetilde{W} 8)$ are satisfied for the event $\widetilde{W}(e, \alpha, T, l-1)$. Thus,

$$
F_{1}(e, \alpha, T, l) \subseteq \widetilde{W}(e, \alpha, T, l-1)
$$

By the induction assumption,

$$
\operatorname{Pr}(\widetilde{W}(e, \alpha, T, l-1)) \leq \varepsilon D^{-y}\left(\frac{r}{\ln r}\right)^{(n-l+1) /(n+1)} .
$$


Let $Z(e, l)$ be the event that $I(v)>s l$ for each $v \in e-M(e, l-1)$. If $\widetilde{W}(e, \alpha, T, l) \cap \overline{X(T)}$ holds, then by $(\widetilde{W} 8), Z(e, l)$ occurs. Thus

$$
F_{1}(e, \alpha, T, l) \subseteq Z(e, l) .
$$

Since we already know whether the event $\widetilde{W}(e, \alpha, T, l-1)$ occurs or not after Step $s(l-1)$, for each $v \in e-M(e, l-1)$,

$$
\begin{aligned}
\operatorname{Pr}(\{I(v)>s l \mid \widetilde{W}(e, \alpha, T, l-1)\}) & \leq \frac{\operatorname{Pr}(I(v)>s l)}{\operatorname{Pr}(I(v)>s(l-1))} \\
& =\frac{1-l p s}{1-(l-1) p s} .
\end{aligned}
$$

Since all random variables $I(v)$ are mutually independent,

$$
\begin{aligned}
\operatorname{Pr}(\{Z(e, l) \mid \widetilde{W}(e, \alpha, T, l-1)\}) & \leq\left(\frac{1-l p s}{1-(l-1) p s}\right)^{r-|M(e, l-1)|} \\
& \leq(1-p s)^{r-|M(e, l-1)|} .
\end{aligned}
$$

Therefore,

$$
\begin{aligned}
& \operatorname{Pr}(\{Z(e, l) \mid \widetilde{W}(e, \alpha, T, l-1)\}) \\
\leq & \sum_{M \subset e} \operatorname{Pr}\{M=M(e, l-1)\}(1-p s)^{r-|M|} .
\end{aligned}
$$

By Lemma $2, \operatorname{Pr}(|M(e, l-1)| \geq z) \leq \varepsilon^{0.5 r}$. Hence

$$
\begin{aligned}
& \sum_{M \subseteq e} \operatorname{Pr}\{M=M(e, l-1)\}(1-p s)^{r-|M|} \\
\leq & \varepsilon^{0.5 r}+\sum_{M \subseteq e:|M|<z} \operatorname{Pr}\{M=M(e, l-1)\}(1-p s)^{r-|M|} \\
\leq & \varepsilon^{0.5 r}+(1-p s)^{r-z} \leq \varepsilon^{0.5 r}+\exp \left\{-p s r\left(1-\frac{4 k^{2}}{\ln r}\right)\right\} .
\end{aligned}
$$

Since $p s \geq \frac{\ln r}{(n+1) r}-p$, by the definition of $p$ and $s$,

$$
\begin{aligned}
\exp \left\{-p s r\left(1-\frac{4 k^{2}}{\ln r}\right)\right\} & \leq \exp \left\{-\left(\frac{\ln r}{n+1}-p r\right)\left(1-\frac{4 k^{2}}{\ln r}\right)\right\} \\
& \leq \exp \left\{-\frac{\ln r}{n+1}+\frac{4 k^{2}}{n+1}+p r\right\} .
\end{aligned}
$$


Recall that $4 k^{2}=-\ln \varepsilon$. Since $p<2^{-k^{r} r}, p r<\frac{4 k^{2}}{n+1}$ and hence

$$
\exp \left\{-\frac{\ln r}{n+1}+\frac{4 k^{2}}{n+1}+p r\right\} \leq r^{\frac{-1}{n+1}} e^{8 k^{2} /(n+1)} \leq \frac{1}{\varepsilon} r^{\frac{-1}{n+1}}
$$

By (3), $\ln r \geq 2 \varepsilon^{-2(n+1)}$ and by (4), $\varepsilon=\exp \left\{-4 k^{2}\right\}$. So,

$$
\frac{1}{\varepsilon} r^{\frac{-1}{n+1}} \leq \frac{1}{\varepsilon}\left(\frac{r}{\ln r}\right)^{\frac{-1}{n+1}} \varepsilon^{2}=\left(\frac{r}{\ln r}\right)^{\frac{-1}{n+1}} \exp \left\{-4 k^{2}\right\}<0.1\left(\frac{r}{\ln r}\right)^{\frac{-1}{n+1}}
$$

By (14) and (16),

$$
\operatorname{Pr}\left(F_{1}(e, \alpha, T, l)\right) \leq \operatorname{Pr}(Z(e, l) \cap \widetilde{W}(e, \alpha, T, l-1)) .
$$

Thus by (15) and (18),

$$
\begin{aligned}
& \operatorname{Pr}\left(F_{1}(e, \alpha, T, l)\right) \\
\leq & \operatorname{Pr}(\{Z(e, l) \mid \widetilde{W}(e, \alpha, T, l-1)\}) \operatorname{Pr}(\widetilde{W}(e, \alpha, T, l-1)) \\
\leq & \left(\varepsilon^{0.5 r}+0.1\left(\frac{r}{\ln r}\right)^{\frac{-1}{n+1}}\right) \operatorname{Pr}(\widetilde{W}(e, \alpha, T, l-1)) \\
\leq & \varepsilon^{0.5 r}+0.1 \varepsilon D^{-y}\left(\frac{r}{\ln r}\right)^{\frac{n-l}{n+1}} .
\end{aligned}
$$

Since by (8), $\varepsilon^{0.5 r-1}<0.03 D^{-k} \leq 0.03 D^{-y}$, this implies (13) for $i=1$.

Now we will prove (13) for $i=2$. Suppose that $F_{2}(e, \alpha, T, l)$ occurs.

- Then, by definition, $\vec{\ell}=\left(l_{1}, \ldots, l_{q}\right) \in \Theta_{2}=\Theta_{2}(q, l)$. In particular, there exists $j^{*} \in[q]$ such that $l_{j^{*}}=l$.

- By $(\widetilde{W} 5)$, for every $j \in[q]$, the event $\widetilde{W}\left(e_{j}, \alpha-2^{l_{j}-1}, T\left(e_{j}\right), l_{j}-1\right)$ occurs.

- Also, for every $j \in[q]$, there exists $h_{j} \in[s]$ such that $I\left(v_{j}\right)=$ $s\left(l_{j}-1\right)+h_{j}$.

- By $(\widetilde{W} 6)$, for every $j \in[q]$, for every $u \in e_{j}$ with $I(u)>\left(l_{j}-1\right) s$, we have also $I(u) \geq\left(l_{j}-1\right) s+h_{j}$.

- By $(\widetilde{W} 1)$ and $(\widetilde{W} 2)$, coloring $\phi(e)$ belongs to the set $\Psi\left(l_{1}, \ldots, l_{q}\right)$ of colorings $\psi$ of $e$ such that the following two properties hold:

(P1) $\psi\left(v_{j}\right)=\alpha-2^{l_{j}-1}$ for all $j \in[q]$. 
(P2) $\psi(v) \in\left\{\psi\left(v_{1}\right), \ldots, \psi\left(v_{q}\right), \alpha\right\}$ for all $v \in e$.

For $j \in[q]$, let $A_{j}=\left\{v \in e: \phi(v)=\phi\left(v_{j}\right)\right\}$ and $a_{j}=\left|A_{j}\right|-1$.

- By $(\widetilde{W} 7)$, for every $j \in[q]$, for each $u \in A_{j}-v_{j},\left(l_{j}-1\right) s+1 \leq I(u) \leq$ $\left(l_{j}-1\right) s+h_{j}$.

Thus, in order for $F_{2}(e, \alpha, T, l)$ to occur, all of the following events should occur:

(R1) There exists a vector $\vec{\ell}=\left(l_{1}, \ldots, l_{q}\right) \in \Theta_{2}=\Theta_{2}(q, l)$ such that for every $j \in[q]$, the event $R_{1}\left(j, l_{j}\right):=\widetilde{W}\left(e_{j}, \alpha-2^{l_{j}-1}, T\left(e_{j}\right), l_{j}-1\right)$ occurs. Let $R_{1}(\vec{\ell}):=\bigcap_{j=1}^{q} R_{1}\left(j, l_{j}\right)$.

(R2) For this vector $\vec{\ell}=\left(l_{1}, \ldots, l_{q}\right)$, there exists a vector $\vec{h}=\left(h_{1}, \ldots, h_{q}\right)$ $\in[s]^{q}$ such that for every $j \in[q]$, the event $R_{2}\left(j, l_{j}, h_{j}\right):=\left\{I\left(v_{j}\right)=\right.$ $\left.s\left(l_{j}-1\right)+h_{j}\right\}$ occurs. Let $R_{2}(\vec{\ell}, \vec{h}):=\bigcap_{j=1}^{q} R_{2}\left(j, l_{j}, h_{j}\right)$.

(R3) For these vectors $\vec{\ell}=\left(l_{1}, \ldots, l_{q}\right)$ and $\vec{h}=\left(h_{1}, \ldots, h_{q}\right)$, for every $j \in[q]$ and every $u \in e_{j}$, the event

$$
R_{3}\left(u, j, l_{j}, h_{j}\right):=\left\{I(u) \notin\left[s\left(l_{j}-1\right)+1, s\left(l_{j}-1\right)+h_{j}-1\right]\right\}
$$

occurs. Let $R_{3}\left(j, l_{j}, h_{j}\right)=\bigcap_{u \in e_{j}-v_{j}} R_{3}\left(u, j, l_{j}, h_{j}\right)$ and $R_{3}(\vec{\ell}, \vec{h}):=$ $\bigcap_{j=1}^{q} R_{3}\left(j, l_{j}, h_{j}\right)$.

(R4) For this vector $\vec{\ell}=\left(l_{1}, \ldots, l_{q}\right)$, there exists a coloring $\psi \in \Psi(\vec{\ell})$ such that the event $R_{4}(\phi, \vec{\ell}):=\{\phi(e)=\psi\}$ occurs.

(R5) For these $\vec{\ell}, \vec{h}$, and $\psi$, if for $j \in[q]$, we define $A_{j}=A_{j}(\psi):=\{v \in$ $\left.e: \psi(v)=\psi\left(v_{j}\right)\right\}$, then for every $j \in[q]$ and $u \in A_{j}$, the event

$$
R_{5}\left(\psi, u, j, l_{j}, h_{j}\right):=\left\{\left(l_{j}-1\right) s+1 \leq I(u) \leq\left(l_{j}-1\right) s+h_{j}\right\}
$$

occurs. Let $R_{5}(\psi, \vec{\ell}, \vec{h}):=\bigcap_{j=1}^{q} \bigcap_{u \in A_{j}-v_{j}} R_{5}\left(\psi, u, j, l_{j}, h_{j}\right)$.

We conclude that

$$
\begin{gathered}
F_{2}(e, \alpha, T, l) \subseteq \bigcup_{\vec{\ell} \in \Theta_{2}} R_{1}(\vec{\ell}) \cap\left[\bigcup _ { \vec { h } \in [ s ] ^ { q } } \left(R_{2}(\vec{\ell}, \vec{h}) \cap R_{3}(\vec{\ell}, \vec{h})\right.\right. \\
\left.\left.\cap \bigcup_{\psi \in \Psi(\vec{\ell})}\left(R_{4}(\psi, \vec{\ell}) \cap R_{5}(\psi, \vec{\ell}, \vec{h})\right)\right)\right] .
\end{gathered}
$$


So, we estimate

$$
\begin{aligned}
& \quad \operatorname{Pr}\left(F_{2}(e, \alpha, T, l)\right) \\
& \leq \sum_{\vec{\ell} \in \Theta_{2}} \operatorname{Pr}\left(R_{1}(\vec{\ell})\right) \sum_{\vec{h} \in[s]^{q}} \operatorname{Pr}\left(\left\{R_{2}(\vec{\ell}, \vec{h}) \cap R_{3}(\vec{\ell}, \vec{h}) \mid R_{1}(\vec{\ell})\right\}\right) \\
& \quad \times \sum_{\psi \in \Psi(\vec{\ell})} \operatorname{Pr}\left(\left\{R_{4}(\psi, \vec{\ell}) \mid R_{1}(\vec{\ell}) \cap R_{2}(\vec{\ell}, \vec{h}) \cap R_{3}(\vec{\ell}, \vec{h})\right\}\right) \\
& \quad \times \operatorname{Pr}\left(\left\{R_{5}(\psi, \vec{\ell}, \vec{h}) \mid R_{1}(\vec{\ell}) \cap R_{2}(\vec{\ell}, \vec{h}) \cap R_{3}(\vec{\ell}, \vec{h}) \cap R_{4}(\psi, \vec{\ell})\right\}\right) .
\end{aligned}
$$

We now will gradually evaluate and simplify the expression in (19). We start from $R_{1}(\vec{\ell})$. Since the vertex sets of $T\left(e_{j}\right)$ for distinct $j$ are disjoint and by Lemma 10 , for every $j$ the event $R_{1}\left(j, l_{j}\right)=\widetilde{W}\left(e_{j}, \alpha-2^{l_{j}-1}, T\left(e_{j}\right), l_{j}-1\right)$ depends only on the values of $I(v)$ and $\phi(v)$ for $v \in V\left(T\left(e_{j}\right)\right)$,

$$
\operatorname{Pr}\left(R_{1}(\vec{\ell})\right)=\prod_{j=1}^{q} \operatorname{Pr}\left(R_{1}\left(j, l_{j}\right)\right)
$$

If $T\left(e_{j}\right)$ has $y_{j}$ edges, then by the induction assumption,

$$
\operatorname{Pr}\left(R_{1}\left(j, l_{j}\right)\right) \leq \varepsilon D^{-y_{j}}\left(\frac{r}{\ln r}\right)^{\left(n-l_{j}+1\right) /(n+1)} .
$$

Thus,

$$
\operatorname{Pr}\left(R_{1}(\vec{\ell})\right) \leq \prod_{j=1}^{q} \varepsilon D^{-y_{j}}\left(\frac{r}{\ln r}\right)^{\left(n-l_{j}+1\right) /(n+1)}
$$

Now, consider the events $R_{3}\left(u, j, l_{j}, h_{j}\right)$. Since all $I(v)$ are independent and all trees $T_{j}$ are vertex-disjoint, $R_{3}\left(u, j, l_{j}, h_{j}\right)$ does not depend on $R_{1}\left(j^{\prime}, l_{j^{\prime}}\right)$ for all $j^{\prime} \neq j$. Furthermore, if $I(u)>s\left(l_{j}-1\right)$, then $\widetilde{W}\left(e_{j}, \alpha-2^{l_{j}-1}, T\left(e_{j}\right), l_{j}-1\right)$ does not depend on the particular value of $I(u)$ in this range. So,

$\operatorname{Pr}\left(\left\{R_{3}\left(u, j, l_{j}, h_{j}\right) \mid R_{1}(\vec{\ell})\right\}\right) \leq \frac{1-p\left(l_{j}-1\right) s-p\left(h_{j}-1\right)}{1-p\left(l_{j}-1\right) s} \leq 1-p\left(h_{j}-1\right)$. 
By the independence of $I(u)$ for distinct $u$, similarly to (17) and the argument following (17), we have

$$
\begin{aligned}
& \operatorname{Pr}\left(\left\{R_{3}\left(j, l_{j}, h_{j}\right) \mid R_{1}(\vec{\ell})\right\}\right) \\
\leq & \sum_{M \subseteq e_{j}} \operatorname{Pr}\left\{M=M\left(e_{j}, l_{j}-1\right)\right\}\left(1-p\left(h_{j}-1\right)\right)^{r-|M|-1} \leq \varepsilon^{0.5 r} \\
& +\sum_{M \subseteq e:|M|<z} \operatorname{Pr}\left\{M=M\left(h_{j}-1\right)\right\}\left(1-p\left(h_{j}-1\right)\right)^{r-|M|-1} \\
\leq & \varepsilon^{0.5 r}+\left(1-p\left(h_{j}-1\right)\right)^{r-z} .
\end{aligned}
$$

Since $\varepsilon^{0.5 r} \leq 0.1(1-p s)^{r} \leq 0.1\left(1-p\left(h_{j}-1\right)\right)^{r-z}$, again by the independence of $I(u)$ for distinct $u$, we conclude that

$$
\operatorname{Pr}\left(\left\{R_{3}(\vec{\ell}, \vec{h}) \mid R_{1}(\vec{\ell})\right\}\right) \leq \prod_{j=1}^{q} 1.1\left(1-p\left(h_{j}-1\right)\right)^{r-z}
$$

Now, we consider events $R_{2}\left(j, l_{j}, h_{j}\right)=\left\{I\left(v_{j}\right)=s\left(l_{j}-1\right)+h_{j}\right\}$. Let $j \in[q]$. Similarly to $R_{3}\left(u, j, l_{j}, h_{j}\right), R_{2}\left(j, l_{j}, h_{j}\right)$ does not depend on $R_{1}\left(j^{\prime}, l_{j^{\prime}}\right)$ for all $j^{\prime} \neq j$, and

$$
\operatorname{Pr}\left(\left\{R_{2}\left(j, l_{j}, h_{j}\right) \mid R_{1}(\vec{\ell})\right\}\right) \leq \frac{p}{1-p s\left(l_{j}-1\right)} \leq p\left(1+\frac{\ln r}{r}\right) .
$$

Moreover, again by the independence of $I(u)$ for distinct $u, R_{2}\left(j, l_{j}, h_{j}\right)$ is independent of all other $R_{2}\left(j^{\prime}, l_{j^{\prime}}, h_{j^{\prime}}\right)$ and of $R_{3}(\vec{\ell}, \vec{h})$. Thus, since $q \leq k \ll$ $\ln r$,

$$
\operatorname{Pr}\left(\left\{R_{2}(\vec{\ell}, \vec{h}) \mid R_{1}(\vec{\ell}) \cap R_{3}(\vec{\ell}, \vec{h})\right\}\right) \leq p^{q}\left(1+\frac{\ln r}{r}\right)^{q} \leq 2 p^{q},
$$

and together with (21), we obtain

$$
\operatorname{Pr}\left(\left\{R_{2}(\vec{\ell}, \vec{h}) \cap R_{3}(\vec{\ell}, \vec{h}) \mid R_{1}(\vec{\ell})\right\}\right) \leq 2 p^{q} \prod_{j=1}^{q} 1.1\left(1-p\left(h_{j}-1\right)\right)^{r-z}
$$

Now for given $\vec{\ell}, \vec{h}$, and $\psi \in \Psi(\vec{\ell})$, we evaluate

$$
\widetilde{P}_{4}(\psi, \vec{\ell}, \vec{h}):=\operatorname{Pr}\left(\left\{R_{4}(\psi, \vec{\ell}) \mid R_{1}(\vec{\ell}) \cap R_{2}(\vec{\ell}, \vec{h}) \cap R_{3}(\vec{\ell}, \vec{h})\right\}\right)
$$


Observe that each event $R_{1}(\vec{\ell}) \cap R_{2}(\vec{\ell}, \vec{h}) \cap R_{3}(\vec{\ell}, \vec{h})$ already fixes the colors of $v_{1}, \ldots, v_{q}$ in $\phi$, but all other vertices of $e$ are "free". Since for each $v \in e$, the value of $\phi(v)$ is chosen independently of all other vertices, for each $\vec{\ell}, \vec{h}$, and $\psi \in \Psi(\vec{\ell})$, we have

$$
\widetilde{P}_{4}(\psi, \vec{\ell}, \vec{h}) \leq k^{q-r} .
$$

Finally, for given $\vec{\ell}, \vec{h}$, and $\psi \in \Psi(\vec{\ell})$, consider the event

$$
\widetilde{R}_{5}(\psi, \vec{\ell}, \vec{h}):=\left\{R_{5}(\psi, \vec{\ell}, \vec{h}) \mid R_{1}(\vec{\ell}) \cap R_{2}(\vec{\ell}, \vec{h}) \cap R_{3}(\vec{\ell}, \vec{h}) \cap R_{4}(\psi, \vec{\ell})\right\} .
$$

For $j \in[q]$, let let $A_{j}=A_{j}(\psi):=\left\{v \in e: \psi(v)=\psi\left(v_{j}\right)\right\}$ and $a_{j}=\left|A_{j}\right|-1$. By definition, for every $j \in[q]$ and every $u \in A_{j}-v_{j}$, the probability of the event

$$
R_{5}\left(\psi, u, j, l_{j}, h_{j}\right):=\left\{\left(l_{j}-1\right) s+1 \leq I(u) \leq\left(l_{j}-1\right) s+h_{j}\right\}
$$

is at most $p h_{j}$. Since the values of $I(v)$ for $v \in e-\left\{v_{1}, \ldots, v_{q}\right\}$ do not depend on $R_{1}(\vec{\ell}) \cap R_{2}(\vec{\ell}, \vec{h}) \cap R_{3}(\vec{\ell}, \vec{h}) \cap R_{4}(\psi, \vec{\ell})$, and are independent of each other for distinct $v$,

$$
\operatorname{Pr}\left(\widetilde{R}_{5}(\psi, \vec{\ell}, \vec{h})\right) \leq \prod_{j=1}^{q}\left(p h_{j}\right)^{a_{j}} .
$$

Since each $\psi \in \Psi\left(l_{1}, \ldots, l_{q}\right)$ is completely defined when we choose disjoint sets $A_{1}-v_{1}, \ldots, A_{q}-v_{q}$ in $e-\left\{v_{1}, \ldots, v_{q}\right\}$,

$$
\begin{aligned}
& \sum_{\psi \in \Psi(\vec{\ell})} \widetilde{P}_{4}(\psi, \vec{\ell}, \vec{h}) \operatorname{Pr}\left(\widetilde{R}_{5}(\psi, \vec{\ell}, \vec{h})\right) \\
\leq & \sum_{a_{1}=0}^{r} \sum_{a_{2}=0}^{r} \cdots \sum_{a_{q}=0}^{r}\left(\begin{array}{c}
r \\
a_{1}
\end{array}\right)\left(\begin{array}{c}
r \\
a_{2}
\end{array}\right) \cdots\left(\begin{array}{c}
r \\
a_{q}
\end{array}\right) k^{q-r} \prod_{j=1}^{q}\left(p h_{j}\right)^{a_{j}} .
\end{aligned}
$$

Thus plugging (20), (23), and (25) into (19), we have

$$
\begin{aligned}
& \operatorname{Pr}\left(F_{2}(e, \alpha, T, l)\right) \leq \sum_{\vec{\ell} \in \Theta_{2}}\left(\prod_{j=1}^{q} \varepsilon D^{-y_{j}}\left(\frac{r}{\ln r}\right)^{\left(n-l_{j}+1\right) /(n+1)}\right) \\
& \times \sum_{\vec{h} \in[s]^{q}} 2 p^{q}\left(\prod_{j=1}^{q} 1.1\left(1-p\left(h_{j}-1\right)\right)^{r-z}\right) \\
& \times \sum_{a_{1}=0}^{r} \sum_{a_{2}=0}^{r} \cdots \sum_{a_{q}=0}^{r}\left(\begin{array}{c}
r \\
a_{1}
\end{array}\right)\left(\begin{array}{c}
r \\
a_{2}
\end{array}\right) \cdots\left(\begin{array}{c}
r \\
a_{q}
\end{array}\right) k^{q-r}\left(p h_{j}\right)^{a_{j}} .
\end{aligned}
$$


We now will simplify and estimate the expression in (27). First observe that

$$
\left(1-p\left(h_{j}-1\right)\right)^{r-z} \leq(1-p)^{(r-z)\left(h_{j}-1\right)} .
$$

Thus since $0<p<2^{-k^{r} r}$ and $h_{j} \leq s \leq \frac{\ln r}{p(n+1) r}$, we have

$$
1.1\left(1-p\left(h_{j}-1\right)\right)^{r-z} \leq 1.1(1-p)^{(r-z)\left(h_{j}-1\right)} \leq 1.2(1-p)^{(r-z) h_{j}} .
$$

So, the expression in (27) is at most

$$
\begin{aligned}
& 2 \sum_{\vec{h} \in[s]^{q}} p^{q}\left(\prod_{j=1}^{q} 1.2(1-p)^{(r-z) h_{j}}\right) \\
& \quad \times \sum_{a_{1}=0}^{r} \sum_{a_{2}=0}^{r} \cdots \sum_{a_{q}=0}^{r}\left(\begin{array}{c}
r \\
a_{1}
\end{array}\right)\left(\begin{array}{c}
r \\
a_{2}
\end{array}\right) \cdots\left(\begin{array}{c}
r \\
a_{q}
\end{array}\right) k^{q-r}\left(p h_{j}\right)^{a_{j}} \\
& =2 k^{-r} \sum_{h_{1}=1}^{s} \sum_{a_{1}=0}^{r} 1.2 p(1-p)^{(r-z) h_{1}}\left(\begin{array}{c}
r \\
a_{1}
\end{array}\right) k\left(p h_{1}\right)^{a_{1}} \\
& \quad \times\left[\sum_{h_{2}=1}^{s} \sum_{a_{2}=0}^{r} 1.2 p(1-p)^{(r-z) h_{2}}\left(\begin{array}{c}
r \\
a_{2}
\end{array}\right) k\left(p h_{2}\right)^{a_{2}}\right. \\
& \left.\times\left[\ldots \sum_{h_{q}=1}^{s} \sum_{a_{q}=0}^{r} 1.2 p(1-p)^{(r-z) h_{q}}\left(\begin{array}{c}
r \\
a_{q}
\end{array}\right) k\left(p h_{q}\right)^{a_{q}}\right] \ldots\right] .
\end{aligned}
$$

For $j=q, q-1, \ldots, 1$ (in this order), we can estimate

$$
\begin{aligned}
& \sum_{h_{j}=1}^{s} \sum_{a_{j}=0}^{r} 1.2 p(1-p)^{(r-z) h_{j}}\left(\begin{array}{c}
r \\
a_{j}
\end{array}\right) k\left(p h_{j}\right)^{a_{j}} \\
\leq & 1.2 p k \sum_{h_{j}=1}^{s}(1-p)^{r h_{j}-z s} \sum_{a_{j}=0}^{r}\left(\begin{array}{c}
r \\
a_{j}
\end{array}\right)\left(p h_{j}\right)^{a_{j}} \\
\leq & 1.2 p k(1-p)^{-z s} \sum_{h_{j}=1}^{s}(1-p)^{r h_{j}}\left(1+p h_{j}\right)^{r} \\
\leq & 1.2 p k e^{\frac{p z s}{1-p}} \sum_{h_{j}=1}^{s}(1-p)^{r h_{j}}\left(1+p h_{j}\right)^{r}
\end{aligned}
$$




$$
\begin{aligned}
& \leq 1.2 p k e^{\frac{z \ln r}{(1-p)(n+1) r}} \sum_{h_{j}=1}^{s}(1-p)^{r h_{j}}(1+p)^{r h_{j}} \\
& <1.2(p s) k e^{\frac{z \ln r}{(1-p)(n+1) r}} \leq 1.2(p s) k e^{\frac{4 k^{2}}{(1-p)(n+1)}} .
\end{aligned}
$$

Since $p s \leq \frac{\ln r}{r(n+1)}, n+1 \geq 2$, and $4 k^{2}=-\ln \varepsilon$, we have

$$
1.2(p s) k e^{\frac{4 k^{2}}{(1-p)(n+1)}} \leq 1.2 \frac{\ln r}{r(n+1)} k \varepsilon^{-1 /(1-p)(n+1)}<e^{-3 k^{2} / 2} \frac{k \ln r}{2 r(n+1) \varepsilon} .
$$

Plugging these bounds into (28)-(29), we obtain that the expression in (27) does not exceed

$$
2 k^{-r} \prod_{j=1}^{q}\left(e^{-3 k^{2} / 2} \frac{k \ln r}{2 r(n+1) \varepsilon}\right) \leq k^{-r} \prod_{j=1}^{q}\left(e^{-3 k^{2} / 2} \frac{k \ln r}{r(n+1) \varepsilon}\right) .
$$

Thus, by (26)-(27)

$$
\begin{aligned}
& \operatorname{Pr}\left(F_{2}(e, \alpha, T, l)\right) \\
\leq & \sum_{\vec{\ell} \in \Theta_{2}} k^{-r} \prod_{j=1}^{q}\left(\varepsilon D^{-y_{j}}\left(\frac{r}{\ln r}\right)^{\frac{n-l_{j}+1}{n+1}}\right)\left(e^{-3 k^{2} / 2} \frac{k \ln r}{r(n+1) \varepsilon}\right) .
\end{aligned}
$$

Note that

$$
k^{-r} D^{-y_{1}-\cdots-y_{q}}=k^{-r} D^{-y+1} \leq D^{-y} \varepsilon\left(\frac{r}{\ln r}\right)^{\frac{n}{n+1}} .
$$

Hence (recalling that $D=\varepsilon k^{r}\left(\frac{r}{\ln r}\right)^{\frac{n}{n+1}}$ ), we may rewrite (30) as

$$
\begin{aligned}
& \operatorname{Pr}\left(F_{2}(e, \alpha, T, l)\right) \\
\leq & D^{-y} \varepsilon\left(\frac{r}{\ln r}\right)^{\frac{n}{n+1}} \sum_{\vec{\ell} \in \Theta_{2}} \prod_{j=1}^{q}\left(\varepsilon\left(\frac{r}{\ln r}\right)^{\frac{n-l_{j}+1}{n+1}}\right)\left(e^{-3 k^{2} / 2} \frac{k \ln r}{r(n+1) \varepsilon}\right) .
\end{aligned}
$$

By the definition of $\Theta_{2}$, for every $\vec{\ell} \in \Theta_{2}$ there is $j^{*}$ such that $l_{j^{*}}=l$. For every $j \neq j^{*}$, we estimate

$$
\varepsilon\left(\frac{r}{\ln r}\right)^{\frac{n-l_{j}+1}{n+1}} e^{-3 k^{2} / 2} \frac{k \ln r}{r(n+1) \varepsilon} \leq \frac{k}{n+1} e^{-3 k^{2} / 2}<\frac{1}{n+1} .
$$


For $j=j^{*}$ we will gain more, since $l_{j^{*}}=l$. Namely,

$$
\varepsilon\left(\frac{r}{\ln r}\right)^{\frac{n-l+1}{n+1}} e^{-3 k^{2} / 2} \frac{k \ln r}{r(n+1) \varepsilon} \leq e^{-3 k^{2} / 2} \frac{k}{n+1}\left(\frac{\ln r}{r}\right)^{\frac{l}{n+1}}
$$

Plugging (33) and (34) into (32), we have

$$
\begin{aligned}
& \operatorname{Pr}\left(F_{2}(e, \alpha, T, l)\right) \\
\leq & D^{-y} \varepsilon\left(\frac{r}{\ln r}\right)^{\frac{n}{n+1}} \sum_{\vec{\ell} \in \Theta_{2}}(n+1)^{-q+1}\left(e^{-3 k^{2} / 2} \frac{k}{n+1}\left(\frac{\ln r}{r}\right)^{\frac{l}{n+1}}\right) .
\end{aligned}
$$

Since the summands in the last expression do not depend on the choice of $\vec{\ell} \in \Theta_{2}$ and $\left|\Theta_{2}\right| \leq(l+1)^{q} \leq(n+1)^{q}$, we have

$$
\begin{aligned}
& \operatorname{Pr}\left(F_{2}(e, \alpha, T, l)\right) \\
\leq & D^{-y} \varepsilon\left(\frac{r}{\ln r}\right)^{\frac{n}{n+1}} \frac{\left|\Theta_{2}\right|}{(n+1)^{q-1}}\left(e^{-3 k^{2} / 2} \frac{k}{n+1}\left(\frac{\ln r}{r}\right)^{\frac{l}{n+1}}\right) \leq \\
\leq & D^{-y} \varepsilon\left(\frac{r}{\ln r}\right)^{\frac{n-l}{n+1}} e^{-3 k^{2} / 2} k \leq 0.4 \varepsilon D^{-y}\left(\frac{r}{\ln r}\right)^{\frac{n-l}{n+1}} .
\end{aligned}
$$

This proves (13) for $i=2$ and thus the lemma.

Applying Lemma 11 for $l=n$, we get the following immediate consequence.

Corollary 1. Let $e \in E(G)$ and $\alpha \in[k]$. Let $D:=\varepsilon k^{r}\left(\frac{r}{\ln r}\right)^{\frac{n}{n+1}}$. Let $T$ be a rooted sub-r-tree of $G$ with root $e$. If $T$ has $y$ edges, then

$$
\operatorname{Pr}(\widetilde{W}(e, \alpha, T, n)) \leq \varepsilon D^{-y} .
$$




\section{Proof of Theorem 2}

Recall the following version of the Local Lemma.

Theorem 4 [1]. Let $A_{1}, A_{2}, \ldots, A_{N}$ be any events. Let $S_{1}, S_{2}, \ldots, S_{N}$ be subsets of $[n]$ such that for each $i, A_{i}$ is independent of the events $\left\{A_{j}\right.$ : $\left.j \in\left([N]-S_{i}\right)\right\}$. If there exist numbers $x_{1}, x_{2}, \ldots, x_{N} \in[0,1)$ such that for all $i \in[N], \operatorname{Pr}\left[A_{i}\right] \leq x_{i} \prod_{j \in S_{i}}\left(1-x_{j}\right)$, Then,

$$
\operatorname{Pr}\left[\bigwedge_{i \in[N]} \overline{A_{i}}\right] \geq \prod_{i \in[N]}\left(1-x_{i}\right)>0
$$

Radhakrishnan and Srinivasan used it in the following form.

Lemma 12 [6]. Let $A_{1}, A_{2}, \ldots, A_{N}$ be any events. Let $S_{1}, S_{2}, \ldots, S_{N}$ be subsets of $[N]$ such that for each $i, A_{i}$ is independent of the events $\left\{A_{j}\right.$ : $\left.j \in\left([N]-S_{i}\right)\right\}$. If for all $i \in[N], \operatorname{Pr}\left(A_{i}\right)<\frac{1}{2}$ and $\sum_{j \in S_{i}} \operatorname{Pr}\left(A_{j}\right) \leq \frac{1}{4}$, then $\operatorname{Pr}\left[\bigwedge_{i \in[N]} \overline{A_{i}}\right]>0$

Proof. We show that if the conditions of this lemma hold, then the conditions of Theorem 4 hold for $x_{i}=2 \operatorname{Pr}\left(A_{i}\right), i \in[N]$. Indeed, with so defined $x_{i}$, inequality

$$
\operatorname{Pr}\left[A_{i}\right] \leq x_{i} \prod_{j \in S_{i}}\left(1-x_{j}\right)
$$

follows if $\prod_{j \in S_{i}}\left(1-x_{j}\right) \geq \frac{1}{2}$ holds. Furthermore,

$$
\prod_{j \in S_{i}}\left(1-x_{j}\right) \geq 1-\sum_{j \in S_{i}} x_{j}=1-2 \sum_{j \in S_{i}} \operatorname{Pr}\left(A_{j}\right) \geq \frac{1}{2}\left(\text { since } \sum_{j \in S_{i}} \operatorname{Pr}\left(A_{j}\right) \leq \frac{1}{4}\right) .
$$

Hence by Theorem 4, we have the result.

Lemma 13. Let $0<\varepsilon \leq 4^{-k} k^{-4}$. If $\operatorname{Pr}(\widetilde{W}(e, \alpha, T, n)) \leq \varepsilon D^{-y}$ for every $\alpha \in[k]$, every sub-r-tree $T$ of $G$ with $y \leq k$ edges and for every $e \in E(T)$, then with positive probability, none of these events occurs. 
Proof. Consider the probability space of the outcomes of Evolution. Let the events $A_{1}, \ldots, A_{N}$ be the events $\widetilde{W}(e, \alpha, T, n)$ for all $e \in E(G)$, all $\alpha \in[k]$ and all sub-r-trees $T$ of $G$ containing $e$ with at most $k$ edges. It is enough to verify that the conditions of Lemma 12 hold for our events $A_{1}, \ldots, A_{N}$. Each of the conditions $\operatorname{Pr}(\widetilde{W}(e, \alpha, T, n))<1 / 2$ immediately follows from Corollary 1. By Lemma 10, for the event $A_{i}=\widetilde{W}(e, \alpha, T, n)$, we can take $S_{i}$ equal to the set of all events $\widetilde{W}\left(e^{\prime}, \alpha^{\prime}, T^{\prime}, n\right)$ such that $V\left(T^{\prime}\right) \cap V(T) \neq \emptyset$.

Now, fix an event $A_{i}=\widetilde{W}(e, \alpha, T, n)$, where $T$ has $y$ edges, and estimate $\sum_{j \in S_{i}} \operatorname{Pr}\left(A_{j}\right)$. Let $\widetilde{W}\left(e^{\prime}, \alpha^{\prime}, T^{\prime}, n\right) \in S_{i}$ and suppose that the size of $T^{\prime}$ is $y^{\prime}$. Then some edge $e^{\prime \prime}$ of $T^{\prime}$ intersects $V(T)$ (in particular, $e^{\prime \prime}$ can be an edge of $T$, too). The number of ways to choose an edge that intersects $V(T)$ is at most $D+1$ if $y=1$, and is at most $y D$, if $y>1$. In any case, this number is not greater than $k D$. By Lemma $7, G$ contains at most $(4 D)^{y^{\prime}-1} r$-trees of size $y^{\prime}$ containing edge $e^{\prime \prime}$. In each of such trees, there are $y^{\prime}$ ways to choose a root, $e^{\prime}$, and $k$ ways to choose the color $\alpha^{\prime}$. Since $\operatorname{Pr}\left(\widetilde{W}\left(e^{\prime}, \alpha^{\prime}, T^{\prime}, n\right)\right) \leq \varepsilon D^{-y^{\prime}}$, it follows that

$$
\sum_{j \in S_{i}} \operatorname{Pr}\left(A_{j}\right) \leq \sum_{y^{\prime}=1}^{k}(k D)(4 D)^{y^{\prime}-1} y^{\prime} k\left(\varepsilon D^{-y^{\prime}}\right)=\sum_{y^{\prime}=1}^{k} k^{2} y^{\prime} 4^{y^{\prime}-1} \varepsilon \leq k^{4} 4^{k-1} \varepsilon .
$$

Since $0<\varepsilon \leq 4^{-k} k^{-4}$, the last expression in (35) is at most $1 / 4$. Thus we are done by Lemma 12 .

Now we are ready to complete the proof of the main theorem. Indeed, let $G$ be a hypergraph satisfying the conditions of the theorem. Consider procedure Evolution. By Corollary 1, for each $y$-edge $r$-tree $T$, each edge $e \in E(T)$ and each $\alpha \in[k], \operatorname{Pr}(\widetilde{W}(e, \alpha, T, n)) \leq \varepsilon D^{-y}$. For $k \geq 2$, we have $\varepsilon=\exp \left\{-4 k^{2}\right\}<4^{-k} k^{-4}$. So, by Lemma 13 , with positive probability none of the events $\widetilde{W}(e, \alpha, T, n)$ occurs. It follows that in some outcome of Evolution none of the events $\widetilde{W}(e, \alpha, T, n)$ occurs. By Lemma 9, in this outcome none of the events $W(e, \alpha, T, n)$ occurs. But then the resulting $k$-coloring will be proper. 


\title{
REFERENCES
}

[1] N. Alon and J. Spencer, The Probabilistic Method, Second Edition, Wiley (2000).

[2] P. Erdős and L. Lovász, Problems and results on 3-chromatic hypergraphs and some related questions, in: Infinite and Finite Sets, A. Hajnal et al., editors, Colloq. Math. Soc. J. Bolyai 11, North Holland (Amsterdam, 1975), pp. 609-627.

[3] A. V. Kostochka, Coloring uniform hypergraphs with few colors, Random Structures and Algorithms, 24 (2004), 1-10.

[4] A. Kostochka and M. Kumbhat, Coloring simple uniform hypergraphs with few edges, to appear in Random Structures and Algorithms.

[5] A. Kostochka and V. Rödl, Constructions of sparse uniform hypergraphs with high chromatic number, submitted.

[6] J. Radhakrishnan and A. Srinivasan, Improved bounds and algorithms for hypergraph two-coloring, Random Structures and Algorithms, 16 (2000), 4-32.

\author{
Alexandr V. Kostochka \\ University of Illinois, \\ Urbana, IL, 61801 \\ and \\ Institute of Mathematics, \\ Novosibirsk, \\ 630090, Russia \\ e-mail: kostochk@math.uiuc.edu \\ Vojtěch Rödl \\ Department of Mathematics and \\ Computer Science, \\ Emory University, \\ Atlanta, GA 30322, \\ U.S.A. \\ e-mail: rodl@mathcs.emory.edu
}

Mohit Kumbhat

Department of Mathematics, University of Illinois,

Urbana, IL, 61801,

U.S.A.

e-mail: kumbhat2@uiuc.edu 